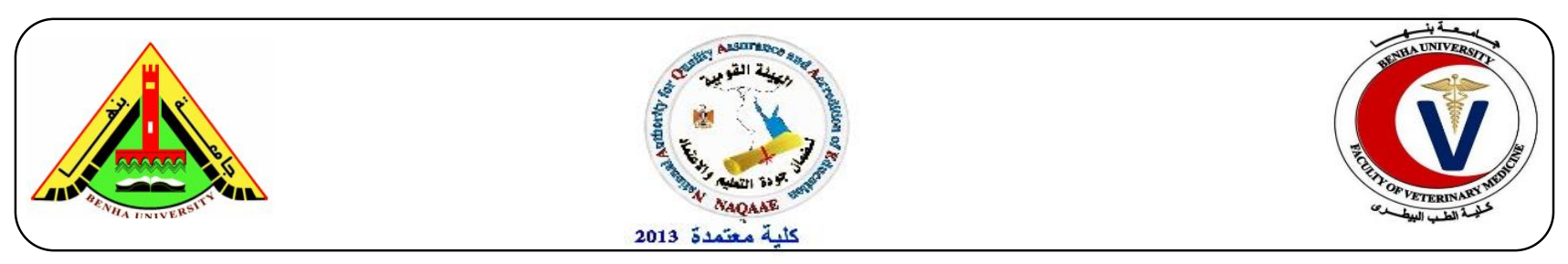

\title{
Effects of chitosan on quality attributes fresh meat slices stored at $4^{0} \mathrm{C}$
}

\author{
Shaltout, F. A. ${ }^{1}$; EL-diasty, E.M. ${ }^{2}$ and Mohamed, M. S. M. ${ }^{3}$ \\ ${ }^{1}$ Food Hygiene Department, Faculty of Veterinary Medicine, Benha University, Egypt. \\ ${ }^{2}$ Mycology Department, Animal Health Research Institute Dokki, Giza. \\ ${ }^{3}$ Animal Health Research, Marsa Matrouh branch.
}

\begin{abstract}
A B S T R A C T
The present study was conducted to evaluate the effect of natural substances as chitosan, thyme oil, lactic acid and acetic acid on microbial, chemical and sensorial quality of fresh meat slices during 18 day of storage at $4^{0} \mathrm{C}$ and periodically analysed for total mould counts, $\mathrm{pH}$, surface colour (Hunter $\mathrm{L}^{*}, \mathrm{a}^{*}$ and $\mathrm{b}^{*}$ values), 2-thiobarbituric acid reactive substance and Total Volatile Nitrogen were measured on days $0,3,6,9,12,15$ and 18 day of storage. The obtained results showed that addition of tested antifungal and antioxidants, individually, affected colour, lipid stability and sensory attributes of the meat slices compared to control during storage. Chitosan $(1.5,1.0$ and $0.5 \% \mathrm{w} / \mathrm{v})$ showed the most intense red colour than other tested antifungal and antioxidants (thyme essential oil, lactic acid and acetic acid) when compared to the control. Also, the results showed that the initial total count (day 0) value for the fresh meat slices was $1 \times 10^{6} \mathrm{CFU} / \mathrm{g}$, then decrease gradually until 9 day not detected any colony by using chitosan $1.5 \%$. While in treated samples with thyme oil $1.5 \%$ and lactic acid $1.5 \%$, the total mould values was $5.6 \times 10^{5} \pm 5.5 \times 10^{3} \mathrm{CFU} / \mathrm{g}$ at 15 days and $9.4 \times 10^{5}$ $\pm 3.9 \times 10^{3} \mathrm{CFU} / \mathrm{g}$ at 12 days. Based on the results obtained in this study, the application of natural substances as chitosan, thyme oil, lactic acid and acetic acid were effective in preserving quality of fresh meat slices and is recommended in meat products.
\end{abstract}

Keywords: Chitosan, Thyme essential oil, Colour, TBA, TVB, Antifungal.

(http://www.bvmj.bu.edu.eg)

(BVMJ-35(2): 157-168, 2018)

\section{INTRODUCTION}

Microbial growth is generally responsible for the spoilage in meats and meat products together with biochemical and enzymatic deteriorations (Devlieghere et al., 2004). In fact, yeasts and fungi contamination is one of the main factors determining the loss in fresh meat quality, since these products are very liable to be contaminated with microorganisms if they are not properly preserved and handled.
The most commonly used strategy used to extend fresh meat shelf life is the use of antimicrobial and antioxidant additives of synthetic origin, which are being questioned due to an increasing consumer demand for natural, healthy and safe preservatives.

Chitosan is a biocompatible polymer derived from shellfish, as a biological sanitizer arises from reports showing several beneficial effects such as antimicrobial and antioxidative 
activities in foods (Friedman and Juneja, 2010). The use of chitosan in industry, agriculture and medicine is well described (Rabea et al., 2003; Senel and McClure, 2004 and Friedman and Juneja, 2010).

Thyme essential oil contains more than 60 ingredients, most of which have important antioxidant and antimicrobial properties (Baranauskiene et al., 2003). Thymol, rosmarinic acid and carvacrol are the most important active compounds of thyme essential oil.

Large amounts of food are lost every year due to spoilage by yeasts and fungi (Hassan et al., 2015). So that, preservative agents commonly used include weak organic acids such as acetic, lactic, benzoic and citric acids, which inhibit the microbial growth in various foods. The effect of organic acids on the fungal growth, which contaminate food and feed, has been investigated by several authors.

Therefore, the aim of our research was a trial to study effect of chitosan, thyme essential oil and organic acids in meat preservation, including fungal growth, chemical, sensory and colour qualities.

\section{Materials and methods}

\subsection{Preparation of spore suspension of $A$.} flavus:

The present study was carried out using a strain of Aspergillus flavus (GenBank accession number: KP137699) isolated previously at the Mycology Department - Animal Health Research Institute, Giza, Egypt. The A. flavus was subcultured and grown for 7 days on Czapek yeast extract. A. flavus culture was washed with $10 \mathrm{ml}$ sterile distilled water in $2 \%$ Tween 80 with the aid of glass beads to help in the spore dispersion. The spore suspensions were standardized to $1 \times 10^{6}$ spores $/ \mathrm{ml}$.

2.2. Preparation of antifungal (chitosan, thyme essential oil, lactic acid and acetic acid):
-Stock solution of chitosan (1.5, 1.0 and $0.5 \%$ $\mathrm{w} / \mathrm{v})$ was prepared in $1.0 \%(\mathrm{v} / \mathrm{v})$ acetic acid.

- Stock solution of lactic acid and acetic acid $(1.5,1.0$ and $0.5 \% \mathrm{v} / \mathrm{v})$ were prepared in sterial distilled water.

- Stock solution of thyme essential oil was used as it is $(1.5,1.0$ and $0.5 \% \mathrm{v} / \mathrm{w})$.

\subsection{Antifungal Activity test:}

Lean beef meat were purchased from the butcher's shop at Benha City, Kalyobia governorate, Egypt, transferred directly to the laboratory in an ice box under complete aseptic condition without undue delay. The lean beef was boneless and trimmed of fat. Then, the beef was cutted into slices (weighing about 100 $\mathrm{g}$ with a size of about $5 \times 5 \times 2 \mathrm{~cm}$ for each slice). Fresh lean beef meat slices were divided into three equal groups as following:

1. First group subjected to total mould count (A. flavus).

2. Second group was for organoleptic examination and

3. Third group was for chemical analysis.

First group was divided into 14 subgroup, while Second group and Third group were divided into 13 subgroup, each sample 100g. One $\mathrm{ml}$ of previously prepared spore suspension was added to each $100 \mathrm{~g}$ of beef meat slices and mixed well. Chitosan, thyme essential oil, lactic acid and acetic acid were added to the meat slice groups to achieve final concentrations of $0.5 \%, 1 \%$ and $1.5 \%(\% \mathrm{v} / \mathrm{g})$. The all antifungal were mixed with the meat slice groups for a further 30 seconds to ensure even mixing. All samples with different treatments and controls were packed in polyethylene bags, labelled and stored at $4{ }^{0} \mathrm{C}$. Mycological evaluation and analysis of stored meat slices under examination (total mould and yeast) was conducted every 3 days during storage period.

\section{Determination of total mould count:}

The technique was applied recommended by ISO 6887 all parts, (2017) and ISO 21527-1 :( 2008) 
Determination of $\mathrm{pH}$ : was done according to the technique recommended by Allen et al. (1997)

Determination of $(T B A)(\mathrm{mg} / \mathrm{kg})$ : was adopted according to (E.O.S 63/8, 2006).

Determination of Total Volatile Nitrogen (TVN) ( $m g \%)$ : was carried out according to (E.O.S 63/10, 2006).

Color measurement: was done according to CIE (1976)

Beef meat slices samples were analyzed at Cairo University Research Park (CURP)/ Faculty of Agriculture, for the following traits: Beef meat slices color was measured by Chroma meter (Konica Minolta, model CR 410, Japan) calibrated with a white plate and light trap supplied by the manufacturer. Color was expressed using the CIE L, a, and b color system (CIE, 1976). A total of three spectral readings were taken for each sample. Lightness ( $\left.\mathrm{L}^{*}\right)$ (dark to light), the redness $\left(\mathrm{a}^{*}\right)$ values (reddish to greenish). The yellowness $\left(b^{*}\right)$ values (yellowish to bluish) were estimated.

\section{RESULTS}

The results of the total mould values (A. flavus) of meat slices treated with chitosan, thyme, acetic acid and lactic acid $(0.5 \%, 1 \%$ and $1.5 \%$ ) during storage at refrigerator at $4 \pm 1{ }^{0} \mathrm{C}$ for 15 days are given in Table (1). The initial total count (day 0) value for the fresh meat slices were $1 \times 10^{6} \mathrm{CFU} / \mathrm{g}$, then decrease gradually until 9 day not detected any colony by using chitosan $1.5 \%$. While in treated samples with thyme oil $1.5 \%$ and lactic acid $1.5 \%$ the total mould values (A. flavus) was $5.6 \times 10^{5} \pm 5.5 \times 10^{3} \mathrm{CFU} / \mathrm{g}$ at 15 days and $9.4 \times 10^{5} \pm 3.9 \times 10^{3} \mathrm{CFU} / \mathrm{g}$ at 12 days (Table 2).

The colour parameters of different formulas of meat slices are summarized in Table 4. For $\mathrm{L}^{*}$ value, fresh meat slices with lactic acid $0.5 \%$, $1 \%$ and $1.5 \%$ had the highest value 42.19 , 42.60 and 42.54 compared to the other treatments and control. As regards to changes in $\mathrm{L}^{*}$ values during storage, there was a decreasing trend in samples containing Chitosan $(0.5 \%, 1 \%$ and $1.5 \%)$ had the lowest value 37.62,36.77 and 35.96,respectively. On the other hand, $\mathrm{L}^{*}$ values during storage of samples containing thymol and acetic acid $(0.5 \%, 1 \% \& 1.5 \%)$ showed an increasing trend, which could be due to gradual protein decomposition, leading to increase of light scattering (McDougall ,1983).

Table 2 revealed that the $\mathrm{pH}$ value at zero day ranged from $5.51 \pm 0.11$ in control samples to $4.30 \pm 0.03,4.25 \pm 0.03,4.23 \pm 0.23,5.49 \pm 0.08$, $5.36 \pm 0.07,5.29 \pm 0.08,4.99 \pm 0.01,4.69 \pm 0.22$, $4.40 \pm 0.06, \quad 5.04 \pm 0.07, \quad 4.72 \pm 0.05 \quad$ and $4.45 \pm 0.04$ for Chitosan $0.5 \%$, Chitosan $1 \%$, Chitosan $1.5 \%$, Thymol $0.5 \%$, Thymol $1 \%$, Thymol $1.5 \%$, Acetic acid $0.5 \%$, Acetic acid $1 \%$, Acetic acid $1.5 \%$, Lactic acid $0.5 \%$, Lactic acid $1 \%$ and Lactic acid $1.5 \%$, treated samples, respectively. These $\mathrm{pH}$ values indicated that all previous natural substances significantly $(\mathrm{P}<0.05)$ lower than control samples at zero day.

The TBA test has been used widely to estimate the extent of lipid oxidation. The TBA value in beef slices treated with chitosan and thyme, acetic acid and lactic acid (0.5\%, $1 \%$ and 1.5) were determined and compared during the 18 days of refrigerated storage. As shown in Table (5), lipid oxidation in samples (control samples) was more intense compared to the other treated samples, reaching 1.040, $1.326, \quad 1.637$

and $1.872 \mathrm{mg} / \mathrm{kg}$ on the $9^{\text {th }}, 12^{\text {th }}, 15^{\text {th }}$ and $18^{\text {th }}$ day of refrigerated storage. The greatest decrease was found in samples treated with chitosan $(0.5 \%, 1 \%$ and 1.5$)$ were 0.615 , $0.489,0.339,0.777,0.527,0.461,0.833$, $0.592,0.512,0.892,0.757$ and $0.578 \mathrm{mg} / \mathrm{kg}$ on the $9^{\text {th }}, 12^{\text {th }}, 15^{\text {th }}$ and $18^{\text {th }}$ day of refrigerated storage.

Table (6) showed that the mean values of total volatile basic nitrogen( TVB-N) of meat slices 
treated with chitosan $0.5 \%$,chitosan $1 \%$,chitosan $1.5 \%$,thyme oil $0.5 \%$,thyme oil $1 \%$,thyme oil $1.5 \%$,acetic acid $0.5 \%$,acetic acid $1 \%$,acetic acid $1.5 \%$,lactic acid $0.5 \%$, lactic acid $1 \%$ and lactic acid $1.5 \%$ at $18^{\text {th }}$ day of preservation are within the permissible limits 14.27, 13.20, 12.43, 15.09, 14.18, 13.26, $19.35, \quad 18.22,17.92,19.87,19.49$ and18.65 $\mathrm{mg} / 100 \mathrm{~g}$, respectively. The mean values of total volatile basic nitrogen (TVB-N) increased rapidly with storage time in the control samples $26.44 \mathrm{mg} / 100 \mathrm{~g}$.

Table 1: Design of chitosan, thyme essential oil, lactic acid and acetic acid added to beef meat slices.

\begin{tabular}{|l|l|}
\hline $\begin{array}{l}\text { Treatments } \\
1^{\text {st }} \text { treatment }\end{array}$ & Meat slice Free from anything (control negative). \\
$2^{\text {nd }}$ treatment & Meat slice + A. flavus $10^{6} \mathrm{spore} / \mathrm{ml}$ (control positive) \\
$3^{\text {th }}$ treatment & Meat slice $+0.5 \%$ chitosan + A. flavus $10^{6} \mathrm{spore} / \mathrm{ml}$ \\
$4^{\text {th }}$ treatment & Meat slice $+1 \%$ chitosan + A. flavus $10^{6} \mathrm{spore} / \mathrm{ml}$. \\
$5^{\text {th }}$ treatment & Meat slice $+1.5 \%$ chitosan + A. flavus $10^{6} \mathrm{spore} / \mathrm{ml}$ \\
$6^{\text {th }}$ treatment & Meat slice $+0.5 \%$ thyme oil + A. flavus $10^{6} \mathrm{spore} / \mathrm{ml}$ \\
$7^{\text {th }}$ treatment & Meat slice $+1 \%$ thyme oil + A. flavus $10^{6} \mathrm{spore} / \mathrm{ml}$. \\
$8^{\text {th }}$ treatment & Meat slice $+1.5 \%$ thyme oil + A. flavus $10^{6} \mathrm{spore} / \mathrm{ml}$ \\
$9^{\text {th }}$ treatment & Meat slice $+0.5 \%$ lactic acid + A. flavus $10^{6} \mathrm{spore} / \mathrm{ml}$ \\
$10^{\text {th }}$ treatment & Meat slice $+1 \%$ lactic acid + A. flavus $10^{6} \mathrm{spore} / \mathrm{ml}$. \\
$11^{\text {th }}$ treatment & Meat slice $+1.5 \%$ lactic acid + A. flavus $10^{6} \mathrm{spore} / \mathrm{ml}$ \\
$12^{\text {th }}$ treatment & Meat slice $+0.5 \%$ acetic acid + A. flavus $10^{6} \mathrm{spore} / \mathrm{ml}$ \\
$13^{\text {th }}$ treatment & Meat slice $+1 \%$ acetic acid + A. flavus $10^{6} \mathrm{spore} / \mathrm{ml}$. \\
$14^{\text {th }}$ treatment & Meat slice $+1.5 \%$ acetic acid + A. flavus $10^{6} \mathrm{spore} / \mathrm{ml}$ \\
\hline
\end{tabular}


Table 2: Antifungal activity of various concentration of different treated meat slices during stored at $4 \pm 1{ }^{\circ} \mathrm{C}$.

Table 3: Effect of chitosan and thymol, acetic acid and lactic acid on sensory attributes of treated

\begin{tabular}{|c|c|c|c|c|c|c|c|c|c|c|c|c|c|c|}
\hline \multirow{2}{*}{ 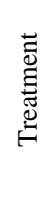 } & \multicolumn{2}{|c|}{ Control } & \multicolumn{3}{|c|}{ Chitosan } & \multicolumn{3}{|c|}{ Thymol } & \multicolumn{3}{|c|}{ Acetic acid } & \multicolumn{3}{|c|}{ Lactic acid } \\
\hline & $\begin{array}{c}\text { Contro } \\
1 \\
(-\mathrm{ve})\end{array}$ & $\begin{array}{c}\text { Contr } \\
\text { ol } \\
(+\mathrm{ve})\end{array}$ & $\begin{array}{c}\text { Chitos } \\
\text { an } \\
0.5 \%\end{array}$ & $\begin{array}{c}\text { Chito } \\
\text { san } \\
1 \%\end{array}$ & $\begin{array}{c}\text { Chitos } \\
\text { an } \\
1.5 \%\end{array}$ & $\begin{array}{c}\text { Thy } \\
\text { mol } \\
0.5 \%\end{array}$ & $\begin{array}{c}\text { Thym } \\
\text { ol } \\
1 \%\end{array}$ & $\begin{array}{c}\text { Thym } \\
\text { ol } \\
1.5 \\
\%\end{array}$ & $\begin{array}{c}\text { Acetic } \\
\text { acid } \\
0.5 \%\end{array}$ & $\begin{array}{c}\text { Acetic } \\
\text { acid } \\
1 \%\end{array}$ & $\begin{array}{c}\text { Acetic } \\
\text { acid } \\
1.5 \%\end{array}$ & $\begin{array}{c}\text { Lactic } \\
\text { acid } \\
0.5 \%\end{array}$ & $\begin{array}{c}\text { Lacti } \\
\text { c acid } \\
1 \%\end{array}$ & $\begin{array}{c}\text { Lactic } \\
\text { acid } \\
1.5 \%\end{array}$ \\
\hline $\begin{array}{c}\text { At } \\
\text { zer } \\
\text { o } \\
\text { da } \\
\text { y }\end{array}$ & $\begin{array}{c}9.7 \times 10 \\
2^{2} \\
5.5 \times 10 \\
1\end{array}$ & $1 \times 10^{6}$ & $1 \times 10^{6}$ & $1 \times 10^{6}$ & $1 \times 10^{6}$ & $\underset{6}{1 \times 10}$ & $1 \times 10^{6}$ & $1 \times 10^{6}$ & $1 \times 10^{6}$ & $1 \times 10^{6}$ & $1 \times 10^{6}$ & $1 \times 10^{6}$ & $1 \times 10^{6}$ & $1 \times 10^{6}$ \\
\hline $3^{\text {th }}$ & $\begin{array}{c}1.4 \times 10 \\
3_{ \pm}^{ \pm} \\
8.5 \times 10 \\
2 \\
\end{array}$ & $\begin{array}{c}1.1 \times 1 \\
0^{6} \pm \\
1.9 \times 1 \\
0^{2} \\
\end{array}$ & $\begin{array}{c}9.6 \times 1 \\
0^{5} \pm \\
3.3 \times 1 \\
0^{2} \\
\end{array}$ & $\begin{array}{c}8.7 \times 1 \\
0^{5} \pm \\
5.5 \times 1 \\
0^{2} \\
\end{array}$ & $\begin{array}{c}3 \times 10^{5} \\
\pm 3.6 \mathrm{x} \\
10^{2}\end{array}$ & $\begin{array}{c}9.9 \mathrm{x} \\
10^{5} \\
\pm 4.2 \\
\times 10^{3} \\
\end{array}$ & $\begin{array}{c}9.6 \mathrm{x} 1 \\
0^{5} \\
\pm 5.2 \mathrm{x} \\
10^{3} \\
\end{array}$ & $\begin{array}{c}6.1 \mathrm{x} 1 \\
0^{5} \\
\pm 9.5 \mathrm{x} \\
10^{3} \\
\end{array}$ & $\begin{array}{c}9.9 \times 10 \\
5 \\
+5 \times 10^{2}\end{array}$ & $\begin{array}{c}9.8 \mathrm{x} 1 \\
0^{5} \\
\pm 6.4 \mathrm{x} \\
10^{2}\end{array}$ & $\begin{array}{c}9.7 \times 1 \\
0^{5} \\
\pm 3.5 \mathrm{x} \\
10^{3}\end{array}$ & $\begin{array}{c}9.9 \times 10 \\
5 \\
\pm 1.7 \times 1 \\
0^{3}\end{array}$ & $\begin{array}{c}9.8 \mathrm{x} 1 \\
0^{5} \\
\pm 6.6 \mathrm{x} \\
10^{3} \\
\end{array}$ & $\begin{array}{c}9.6 \times 10 \\
5 \\
\pm 2.8 \times 1 \\
0^{3} \\
\end{array}$ \\
\hline $6^{\text {th }}$ & $\begin{array}{c}7.3 \times 10 \\
3_{ \pm}^{ \pm} \\
2.7 \times 10 \\
3\end{array}$ & $\begin{array}{c}6.7 \times 1 \\
0^{6} \pm \\
7.9 \times 1 \\
0^{2}\end{array}$ & $\begin{array}{c}8.9 \times 1 \\
0^{5} \pm \\
3.8 \times 1 \\
0^{2}\end{array}$ & $\begin{array}{c}5.2 \mathrm{x} 1 \\
0^{5} \\
\pm 5.9 \mathrm{x} \\
10^{2} \\
\end{array}$ & $\begin{array}{c}9.8 \times 1 \\
0^{4} \pm \\
5.2 \times 1 \\
0^{2}\end{array}$ & $\begin{array}{c}9.8 \mathrm{x} \\
10^{5} \\
\pm 1.9 \\
\mathrm{x} 10^{3}\end{array}$ & $\begin{array}{c}7.6 \mathrm{x} 1 \\
0^{5} \\
\pm 1.2 \mathrm{x} \\
10^{3}\end{array}$ & $\begin{array}{c}4 \times 10^{5} \\
\pm 7.3 \mathrm{x} \\
10^{3}\end{array}$ & $\begin{array}{c}9.9 \times 10 \\
5 \\
\pm 4.4 \times 1 \\
0^{2}\end{array}$ & $\begin{array}{c}9.7 \times 1 \\
0^{5} \\
\pm 7.1 \mathrm{x} \\
10^{3}\end{array}$ & $\begin{array}{c}9.6 \times 1 \\
0^{5} \\
\pm 2.5 \mathrm{x} \\
10^{5}\end{array}$ & $\begin{array}{c}9.9 \times 10 \\
5 \\
\pm 5 \times 10^{3}\end{array}$ & $\begin{array}{c}9.6 \mathrm{x} 1 \\
0^{5} \\
\pm 4.6 \mathrm{x} \\
10^{3}\end{array}$ & $\begin{array}{c}9.5 \times 10 \\
5 \\
\pm 1.4 \times 1 \\
0^{3} \\
\end{array}$ \\
\hline $9^{\text {th }}$ & \multirow{3}{*}{$\begin{array}{l}\frac{7}{0} \\
\overline{0} \\
\text { की }\end{array}$} & \multirow{3}{*}{$\begin{array}{l}\frac{0}{0} \\
\overline{0} \\
\text { की }\end{array}$} & \multirow{3}{*}{$\begin{array}{l}\frac{\overline{0}}{\overline{0}} \\
\frac{0}{2} \\
\text { की }\end{array}$} & $\begin{array}{c}4.4 \times 1 \\
0^{5} \pm \\
2.2 \times 1 \\
0^{2} \\
\end{array}$ & \multirow{3}{*}{ そ } & \multirow{3}{*}{$\begin{array}{l}\frac{\pi}{0} \\
\frac{0}{0} \\
\text { के }\end{array}$} & $\begin{array}{c}6.4 \times 1 \\
0^{5} \\
\pm 1.5 \mathrm{x} \\
10^{3} \\
\end{array}$ & $\begin{array}{c}3.9 \times 1 \\
0^{5} \\
\pm 7.1 \mathrm{x} \\
10^{3} \\
\end{array}$ & \multirow{3}{*}{$\begin{array}{l}\frac{\overline{0}}{\overline{0}} \\
\text { की }\end{array}$} & \multirow{3}{*}{$\begin{array}{l}\frac{\bar{d}}{\overline{0}} \\
\text { की }\end{array}$} & \multirow{3}{*}{$\begin{array}{l}\frac{\overrightarrow{0}}{\pi 0} \\
\text { की }\end{array}$} & \multirow{3}{*}{$\begin{array}{l}\overrightarrow{0} \\
\frac{0}{0} \\
\text { के }\end{array}$} & $\begin{array}{c}9.7 \times 1 \\
0^{5} \\
\pm 1.2 \mathrm{x} \\
10^{3} \\
\end{array}$ & $\begin{array}{c}9.5 \times 10 \\
5 \\
\pm 2.5 \times 1 \\
0^{3} \\
\end{array}$ \\
\hline $\begin{array}{l}12 \\
\text { th }\end{array}$ & & & & \multirow{2}{*}{ 之 } & & & $\begin{array}{c}7 \times 10^{5} \\
\pm 2.5 \mathrm{x} \\
10^{3}\end{array}$ & $\begin{array}{c}4.9 \mathrm{x} 1 \\
0^{5} \\
\pm 2.6 \mathrm{x} \\
10^{3} \\
\end{array}$ & & & & & \multirow{2}{*}{$\begin{array}{l}\frac{\overrightarrow{0}}{\pi 0} \\
\text { की } \\
\text { की }\end{array}$} & $\begin{array}{c}9.4 \times 10 \\
5 \\
\pm 3.9 \times 1 \\
0^{3} \\
\end{array}$ \\
\hline $\begin{array}{l}15 \\
\text { th }\end{array}$ & & & & & & & $\begin{array}{l}\text { Spoil } \\
\text { ed }\end{array}$ & $\begin{array}{c}5.6 \mathrm{x} 1 \\
0^{5} \\
\pm 5.5 \mathrm{x} \\
10^{3} \\
\end{array}$ & & & & & & $\begin{array}{c}\text { Spoile } \\
\text { d }\end{array}$ \\
\hline
\end{tabular}

meat slices during stored at $4 \pm 1{ }^{\circ} \mathrm{C}$.

\begin{tabular}{|c|c|c|c|c|c|c|c|c|c|c|c|c|c|c|c|}
\hline \multirow[t]{2}{*}{ Item } & \multicolumn{3}{|c|}{ control } & \multicolumn{3}{|c|}{ chitosan } & \multicolumn{3}{|c|}{ Thyme oil } & \multicolumn{3}{|c|}{ Lactic acid } & \multicolumn{3}{|c|}{ Acetic acid } \\
\hline & $\begin{array}{c}0.5 \\
\%\end{array}$ & $1 \%$ & $\begin{array}{c}1.5 \\
\%\end{array}$ & $\begin{array}{c}0.5 \\
\%\end{array}$ & $1 \%$ & $1.5 \%$ & $\begin{array}{c}0.5 \\
\%\end{array}$ & $\begin{array}{l}1 \\
\%\end{array}$ & $\begin{array}{c}1.5 \\
\%\end{array}$ & $0.5 \%$ & $1 \%$ & $1.5 \%$ & $\begin{array}{c}0.5 \\
\%\end{array}$ & $\begin{array}{l}1 \\
\%\end{array}$ & $\begin{array}{c}1.5 \\
\%\end{array}$ \\
\hline Color & 10 & 10 & 10 & 3 & 2 & 2 & 9 & 10 & 10 & 10 & 10 & 10 & 10 & 10 & 10 \\
\hline Odor & 2 & 3 & 4 & 10 & 10 & 10 & 10 & 10 & 10 & 10 & 8 & 7 & 10 & 8 & 7 \\
\hline
\end{tabular}

Table 4: Effect of chitosan and thymol, acetic acid and lactic acid on $\mathrm{pH}$ value and Hunter colour values $\left(\mathrm{L}^{*}, \mathrm{a}^{*}\right.$ and $\left.\mathrm{b}^{*}\right)$ of red meat slice during storage at $4 \pm 1{ }^{\circ} \mathrm{C}$ at 3 days.

\begin{tabular}{|l|c|c|c|c|}
\hline \multicolumn{1}{|c|}{ Treatment } & $\mathrm{pH}$ & $\mathrm{L}^{*}$ & $\mathrm{~A}^{*}$ & $\mathrm{~B}^{*}$ \\
\hline Control & $5.72 \pm 0.06$ & $42.07 \pm 0.28$ & $19.63 \pm 0.95$ & $10.48 \pm 0.50$ \\
\hline Chitosan 0.5\% & $4.39 \pm 0.06$ & $37.62 \pm 0.35$ & $8.59 \pm 0.13$ & $6.91 \pm 0.14$ \\
\hline Chitosan 1\% & $4.29 \pm 0.03$ & $36.77 \pm 0.32$ & $8.19 \pm 0.21$ & $6.54 \pm 0.17$ \\
\hline Chitosan 1.5\% & $4.25 \pm 0.03$ & $35.96 \pm 0.19$ & $8.09 \pm 0.05$ & $6.28 \pm 0.09$ \\
\hline Thymol 0.5\% & $5.51 \pm 0.07$ & $41.11 \pm 0.23$ & $19.28 \pm 0.56$ & $9.83 \pm 0.33$ \\
\hline Thymol 1\% & $5.41 \pm 0.06$ & $37.86 \pm 1.45$ & $18.49 \pm 0.73$ & $8.74 \pm 0.35$ \\
\hline Thymol 1.5\% & $5.33 \pm 0.07$ & $37.73 \pm 0.42$ & $17.02 \pm 0.19$ & $8.18 \pm 0.17$ \\
\hline Acetic acid 0.5\% & $5.10 \pm 0.06$ & $41.57 \pm 0.37$ & $19.53 \pm 0.24$ & $10.08 \pm 0.03$ \\
\hline Acetic acid 1\% & $4.97 \pm 0.09$ & $41.49 \pm 0.46$ & $19.55 \pm 0.31$ & $10.17 \pm 0.06$ \\
\hline Acetic acid 1.5\% & $4.86 \pm 0.07$ & $40.17 \pm 0.24$ & $19.04 \pm 0.27$ & $10.06 \pm 0.66$ \\
\hline Lactic acid 0.5\% & $5.20 \pm 0.03$ & $42.19 \pm 0.10$ & $19.57 \pm 1.11$ & $10.43 \pm 0.17$ \\
\hline Lactic acid 1\% & $5.09 \pm 0.10$ & $42.60 \pm 0.43$ & $19.42 \pm 0.14$ & $10.66 \pm 0.36$ \\
\hline Lactic acid 1.5\% & $4.97 \pm 0.11$ & $42.54 \pm 0.62$ & $19.27 \pm 0.46$ & $10.91 \pm 0.26$ \\
\hline
\end{tabular}


Shaltout et al. (2018). BVMJ-35(2): 157-168

Table 5: TBA values of meat slices treated with different treatments during 18 days of refrigerated storage at $4 \pm 1{ }^{\circ} \mathrm{C}(\mathrm{mg} / \mathrm{kg})$.

\begin{tabular}{|c|c|c|c|c|c|c|c|}
\hline Treatment & At zero day & $3^{\text {th }}$ & $6^{\text {th }}$ & $9^{\text {th }}$ & $12^{\text {th }}$ & $15^{\text {th }}$ & $18^{\text {th }}$ \\
\hline Control & $0.041 \pm 0.033$ & $0.72 \pm 0.01$ & $0.95 \pm 0.03$ & $1.040 \pm 0.04$ & $1.326 \pm 0.13$ & $1.637 \pm 0.09$ & $1.872 \pm 0.04$ \\
\hline $\begin{array}{l}\text { Chitosan } \\
0.5 \%\end{array}$ & $0.037 \pm 0.004$ & $0.44 \pm 0.02$ & $0.56 \pm 0.02$ & $0.615 \pm 0.43$ & $0.777 \pm 0.54$ & $0.833 \pm 0.58$ & $0.892 \pm 0.62$ \\
\hline $\begin{array}{l}\text { Chitosan } \\
1 \%\end{array}$ & $0.037 \pm 0.003$ & $0.350 \pm 0.02$ & $0.410 \pm 0.03$ & $0.489 \pm 0.34$ & $0.527 \pm 0.37$ & $0.592 \pm 0.41$ & $0.757 \pm 0.019$ \\
\hline $\begin{array}{l}\text { Chitosan } \\
1.5 \%\end{array}$ & $0.034 \pm 0.024$ & $0.27 \pm 0.02$ & $0.30 \pm 0.02$ & $0.339 \pm 0.24$ & $0.461 \pm 0.32$ & $0.512 \pm 0.36$ & $0.578 \pm 0.401$ \\
\hline $\begin{array}{l}\text { Thymol } \\
0.5 \%\end{array}$ & $0.037 \pm 0.026$ & $0.609 \pm 0.42$ & $0.699 \pm 0.48$ & $0.789 \pm 0.55$ & $0.869 \pm 0.60$ & $1.015 \pm 0.70$ & $1.605 \pm 1.11$ \\
\hline $\begin{array}{l}\text { Thymol } \\
1 \%\end{array}$ & $0.038 \pm 0.027$ & $0.548 \pm 0.38$ & $0.780 \pm 0.54$ & $0.793 \pm 0.55$ & $0.858 \pm 0.60$ & $0.991 \pm 0.69$ & $1.507 \pm 1.04$ \\
\hline $\begin{array}{l}\text { Thymol } \\
1.5 \%\end{array}$ & $0.037 \pm 0.026$ & $0.480 \pm 0.33$ & $0.64 \pm 0.44$ & $0.723 \pm 0.50$ & $0.802 \pm 0.56$ & $0.904 \pm 0.63$ & $1.322 \pm 0.92$ \\
\hline $\begin{array}{l}\text { Acetic acid } \\
0.5 \%\end{array}$ & $0.039 \pm 0.27$ & $0.640 \pm 0.44$ & $0.840 \pm 0.53$ & $0.891 \pm 0.62$ & $0.963 \pm 0.67$ & $1.224 \pm 0.85$ & $1.646 \pm 1.14$ \\
\hline $\begin{array}{l}\text { Acetic acid } \\
1 \%\end{array}$ & $0.037 \pm 0.026$ & $0.587 \pm 0.41$ & $0.813 \pm 0.56$ & $0.925 \pm 0.64$ & $1.178 \pm 0.82$ & $1.350 \pm 0.94$ & $1.530 \pm 1.06$ \\
\hline $\begin{array}{l}\text { Acetic acid } \\
1.5 \%\end{array}$ & $0.036 \pm 0.025$ & $0.530 \pm 0.37$ & $0.792 \pm 0.55$ & $0.931 \pm 0.65$ & $1.041 \pm 0.72$ & $1.408 \pm 0.98$ & $1.592 \pm 1.10$ \\
\hline $\begin{array}{l}\text { Lactic acid } \\
0.5 \%\end{array}$ & $0.044 \pm 0.631$ & $0.646 \pm 0.45$ & $0.904 \pm 0.63$ & $1.204 \pm 0.84$ & $1.434 \pm 0.99$ & $1.655 \pm 1.15$ & $1.839 \pm 0.14$ \\
\hline $\begin{array}{l}\text { Lactic acid } \\
1 \%\end{array}$ & $0.040 \pm 0.028$ & $0.590 \pm 0.41$ & $0.805 \pm 0.56$ & $0.983 \pm 0.68$ & $1.378 \pm 0.96$ & $1.512 \pm 1.05$ & $1.795 \pm 1.25$ \\
\hline $\begin{array}{l}\text { Lactic acid } \\
1.5 \%\end{array}$ & $0.038 \pm 0.026$ & $0.573 \pm 0.40$ & $0.799 \pm 0.55$ & $0.951 \pm 0.66$ & $1.308 \pm 0.91$ & $1.411 \pm 0.98$ & $1.634 \pm 1.133$ \\
\hline
\end{tabular}

Table 6: TBA values of meat slices treated with different treatments during 18 days of refrigerated storage at $4 \pm 1{ }^{\circ} \mathrm{C}(\mathrm{mg} / \mathrm{kg})$.

\begin{tabular}{|c|c|c|c|c|c|c|c|}
\hline Treatment & $\begin{array}{c}\text { At zero } \\
\text { day }\end{array}$ & $3^{\text {th }}$ & $6^{\text {th }}$ & $9^{\text {th }}$ & $12^{\text {th }}$ & $15^{\text {th }}$ & $18^{\text {th }}$ \\
\hline Control & $6.18 \pm 0.09$ & $17.53 \pm 0.55$ & $19.26 \pm 0.22$ & $20.04 \pm 0.58$ & $21.15 \pm 0.21$ & $24.41 \pm 0.37$ & $26.44 \pm 3.39$ \\
\hline $\begin{array}{l}\text { Chitosan } \\
0.5 \%\end{array}$ & $6.26 \pm 0.13$ & $8.61 \pm 0.18$ & $9.08 \pm 0.08$ & $11.13 \pm 0.13$ & $12.33 \pm 0.06$ & $13.28 \pm 0.35$ & $14.27 \pm 0.12$ \\
\hline Chitosan $1 \%$ & $6.11 \pm 0.58$ & $7.96 \pm 0.30$ & $8.65 \pm 0.23$ & $10.68 \pm 0.17$ & $12.02 \pm 0.24$ & $12.71 \pm 0.43$ & $13.20 \pm 0.12$ \\
\hline $\begin{array}{l}\text { Chitosan } \\
1.5 \%\end{array}$ & $6.18 \pm 0.07$ & $7.41 \pm 0.05$ & $8.02 \pm 0.07$ & $10.08 \pm 0.08$ & $11.43 \pm 0.40$ & $11.65 \pm 0.33$ & $12.43 \pm 0.24$ \\
\hline $\begin{array}{l}\text { Thyme oil } \\
0.5 \%\end{array}$ & $6.11 \pm 0.58$ & $8.87 \pm 0.09$ & $9.43 \pm 0.08$ & $11.70 \pm 0.16$ & $13.78 \pm 0.22$ & $14.03 \pm 0.06$ & $15.09 \pm 0.12$ \\
\hline $\begin{array}{l}\text { Thyme oil } \\
1 \%\end{array}$ & $6.26 \pm 0.13$ & $8.06 \pm 0.07$ & $9.01 \pm 0.05$ & $11.09 \pm 0.27$ & $12.58 \pm 0.32$ & $13.59 \pm 0.10$ & $14.18 \pm 0.12$ \\
\hline $\begin{array}{l}\text { Thyme oil } \\
1.5 \%\end{array}$ & $6.18 \pm 0.09$ & $7.59 \pm 0.19$ & $8.35 \pm 0.03$ & $10.71 \pm 0.20$ & $11.83 \pm 0.31$ & $12.38 \pm 0.14$ & $13.26 \pm 0.02$ \\
\hline $\begin{array}{l}\text { Acetic acid } \\
0.5 \%\end{array}$ & $6.30 \pm 0.10$ & $10.28 \pm 0.34$ & $12.40 \pm 0.07$ & $14.3 \pm 0.42$ & $15.87 \pm 0.20$ & $17.23 \pm 0.15$ & $19.35 \pm 0.05$ \\
\hline $\begin{array}{l}\text { Acetic acid } \\
1 \%\end{array}$ & $6.22 \pm 0.07$ & $9.79 \pm 0.11$ & $11.78 \pm 0.19$ & $14.49 \pm 0.31$ & $15.20 \pm 0.10$ & $16.94 \pm 0.10$ & $18.22 \pm 0.17$ \\
\hline $\begin{array}{l}\text { Acetic acid } \\
1.5 \%\end{array}$ & $6.18 \pm 0.06$ & $9.65 \pm 0.23$ & $11.70 \pm 0.09$ & $13.81 \pm 0.26$ & $15.11 \pm 0.10$ & $16.91 \pm 0.02$ & $17.92 \pm 0.41$ \\
\hline $\begin{array}{l}\text { Lactic acid } \\
0.5 \%\end{array}$ & $6.58 \pm 0.19$ & $11.37 \pm 0.28$ & $13.00 \pm 0.02$ & $14.34 \pm 0.23$ & $16.72 \pm 0.12$ & $18.0 \pm 0.06$ & $19.87 \pm 0.33$ \\
\hline
\end{tabular}


Effects of chitosan on quality attributes fresh meat slices stored at $4^{0} \mathrm{C}$

\begin{tabular}{llllllll}
\hline $\begin{array}{l}\text { Lactic acid } \\
1 \%\end{array}$ & $6.34 \pm 0.04$ & $11.07 \pm 0.14$ & $12.53 \pm 0.10$ & $13.87 \pm 0.03$ & $15.64 \pm 0.19$ & $17.55 \pm 0.29$ & $19.49 \pm 0.27$ \\
$\begin{array}{l}\text { Lactic acid } \\
1.5 \%\end{array}$ & $6.25 \pm 0.05$ & $10.67 \pm 0.33$ & $12.24 \pm 0.36$ & $13.27 \pm 0.16$ & $15.22 \pm 0.34$ & $17.11 \pm 0.55$ & $18.65 \pm 0.29$ \\
\hline
\end{tabular}
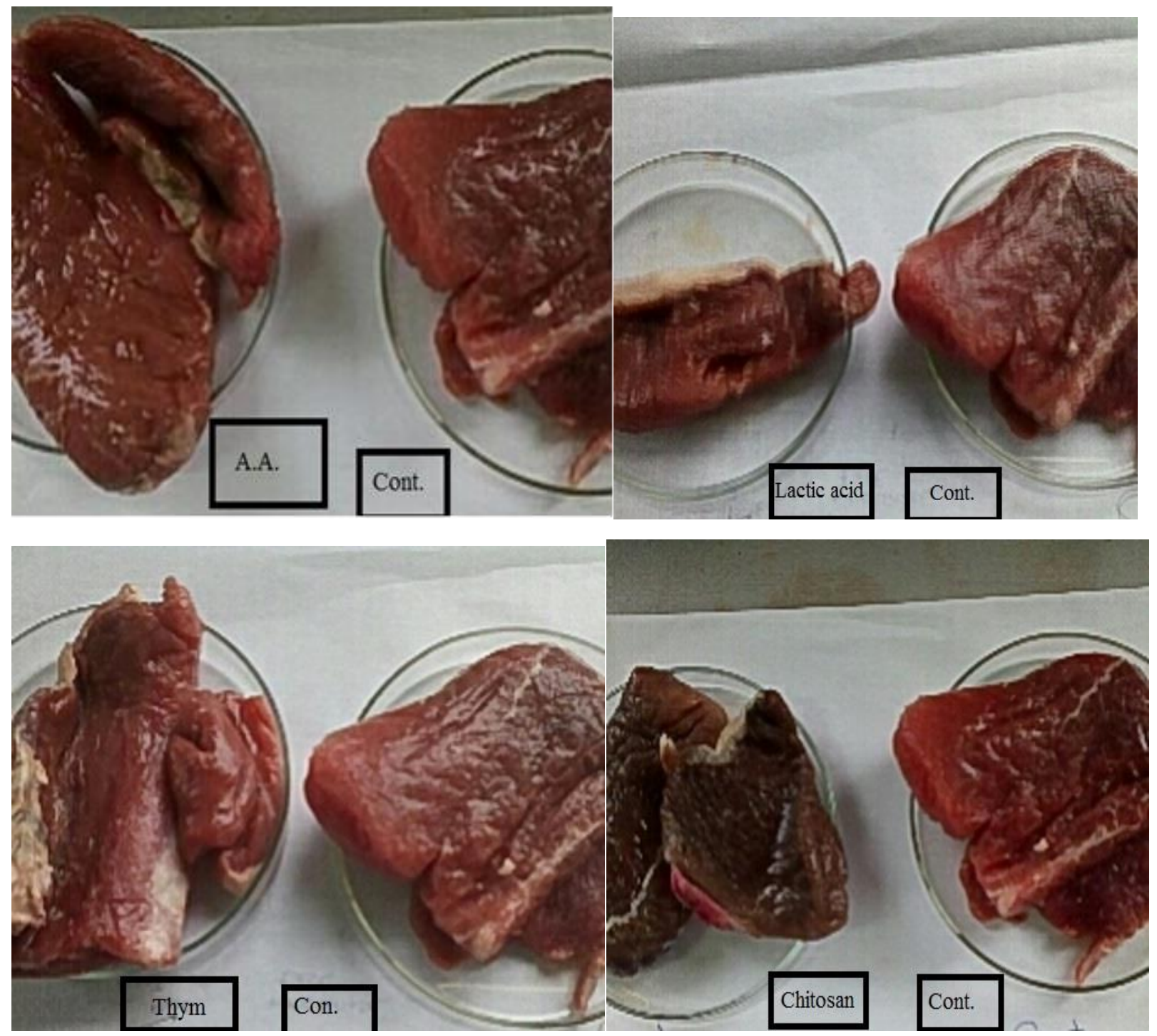

Fig.1. Show meat colour of (a): acetic acid, (b) lactic acid), (c) thyme essential oil and (d) chitosan treatment. 


\section{DISCUSSION}

Meat and especially meat products are highly susceptible to both microbial growth and lipid oxidation because of their large surface to weight ratio, leading to rapid spoilage and development of rancid or warmed-over flavour, respectively (Jay et al., 2005). Because there is an increasing consumer demand for minimally processed foods without chemical preservatives, the food industry is facing a constant challenge to develop alternative 'natural' methods to extend product shelf life and improve safety (Karabagias et al., 2011).

Low molecular weight water-soluble chitosan (LMWS-chitosan) strongly inhibited the growth of $A$. fumigatus, $A$. parasiticus, $B$. cinerea, $F$. solani, $F$. oxysporum, and $P$. verrucosum Park et al. (2008). The antifungic mechanism of chitosan involves cell wall morphogenesis with chitosan molecules interfering directly with fungal growth. Microscopic observation reported that chitosan oligomers diffuse inside hyphae interfering on the enzymes activity responsible for the fungus growth. The intensity of degradation action of chitosan on fungal cell walls is also dependent upon the concentration, degree of acetylation and local pH. Studies conducted on Rhizoctonia solani revealed that the percentage of fungus germination decreased with increasing the chitosan concentration in the medium (Goy et al., 2009).

The possible mechanism of action of essential oil components on the growth of fungi was reported in several studies. It is generally accepted that the essential oil components act on the functionality and the structure of the cell membrane. Low concentrations result in changes of the cell structure, inhibiting respiration and changing the permeability of the cell membrane, whereas high concentrations lead to severe membrane damage, loss of homeostasis and cell death (Kocić-Tanackov and Dimić (2013). Different acetic and lactic acid concentrations (5 and 10 $\%$ ) were studied by (Hassan et al., 2015) for antifungal activity against different strains of A. flavus. They determined that the increase of acid in the medium decreases the growth rate and extends the lag phase, when acetic acid and lactic acid (10\%) were used the inhibition effect were $40.92 \%$ and $17.96 \%$, respectively.

Meat colour is the first condition that consumer use to judge meat quality and acceptability. Anwer et al. (2013) reported that colour is an important factor in selection of meat products. Meat colour is one of the most important for consumers are indication of an originality and uprightness. Consumers will often refuse products in which the colour varies from the predictable appearance. Therefore, colour is frequently used to determine economic value of food.

In organoleptic examination the test revealed that, (as shown in Table 3,Fig 1) the colour of the control sample not changed, while the odour slightly changed in concentration $1.5 \%$ (score 4) after 3 days of preservation. In samples treated with chitosan and thymol, acetic acid and lactic acid $(0.5 \%, 1 \%$ and $1.5 \%)$, the colour changed into dark red after preservation only in case of chitosan $(0.5 \%, 1 \%$ and $1.5 \%$ ) score 3,2 and 2 , respectively. The odour slightly changed after preservation dilution $1 \%$ and $1.5 \%$ of lactic acid and acetic acid (score 8 and 7), respectively.

The $\mathrm{pH}$ value of all meat slices samples slightly decreased in chitosan than other treatments during the storage, this decrease indicates that some fermentation occurs during storage (Table 4,Fig 1). This result agree with Mokhtar et al. (2014) where reported that the $\mathrm{pH}$ value slightly decreased during the first 3 days of storage, whereas after day 3 there was a gradual increase. This decrease indicates 
that some fermentation occurs during storage. The last $\mathrm{pH}$ values increase might have been due to the liberation of ammonia compounds as a result of endprotease activity or the proteolytic microbial flora present in the raw meat Mokhtar et al., (2012).

Regarding to The changes in $\mathrm{L}^{*}$ values during the storage period, there was a significant increase in samples dipped in $1 \%$ chitosan (CS) compared with chitosan oligosaccharides (COS) (chitosan subjected to $\gamma$ irradiation, 50 or $100 \mathrm{kGy}$ ) Zahran (2015). Also similar trend was reported by Jo et al. (2001) which could be the result of gradual protein decomposition, leading to the increase of light scattering.

Further $\mathrm{L}^{*}$ values in meat and meat products are related to surface water, water vapour exchanges between the products and the environment and modifications of the different states of the heme pigments Fernández-López et al. (2000).

The values of meat slices were affected by the addition of the examined preservatives. Samples containing chitosan had a lower a* values than samples containing lactic acid, thymol and acetic acid throughout the whole period of storage. Similar results were observed by Georgantelis et al. (2007) and Mokhtar et al. (2014) who found that changes in $\mathrm{L}^{*}$ values during storage, there was a decreasing trend in samples containing Chitosan and a decreasing trend was observed as regards to $\mathrm{a}^{*}$ values, which is attributed to the gradual oxidation of myoglobin, conversion of myoglobin into metmyoglobin and accumulation of metmyoglobin with time. A significant decrease in chrome values (brownish colour development), which could be a drawback in terms of consumer acceptance.

The $\mathrm{b}^{*}$ values of chitosan-treated samples $(0.5 \%, 1 \%$ and $1.5 \%)$ were decrease than samples containing lactic acid, acetic acid and thymol, which could be attributed to the natural yellowish colour of chitosan affecting the meat slices colour. Similar observations have been also found by Georgantelis et al. (2007) in beef burger, Jo et al., (2001) and Youn et al., (1999) in pork sausage with added chitosan. During refrigerated storage, a decreasing trend in the control and all treated samples was observed. Zahran (2015) reported that initial measurements (day-1) of $b^{*}$-values (yellowness) were higher $(\mathrm{p}<0.05)$ in samples dipped in chitosan and chitosan oligomer compared with control and acetic acid 1\%. Also, after 2 weeks of storage, $b^{*}$ - values increased in samples dipped in acetic acid $1 \%$ and chitosan $1 \%$ irradiated $50 \mathrm{kGy} \gamma$, but decreased in control, acetic acid $1 \%$ and chitosan $1 \%$ irradiated $100 \mathrm{kGy} \gamma$.

Beef muscle with greater lipid oxidation accelerated greater protein oxidation and metmyoglobin formation which lead variation in physicochemical quality of beef muscle. Chemical deterioration related to $\mathrm{pH}$ and lipid oxidations have greater effect in declining palatability and texture of beef and beef products. Fresh beef and beef products preserved under refrigeration state can still develop physicochemical deterioration slowly. The degree of lipid oxidations affected by various thawing methods during frozen state is still a potential problem and effective methods need to be explored (Rahman et al., 2015).

The results of TBA are agreement with Georgantelis et al., (2007) who observed lower TBA values for fresh pork treated with chitosan. Also, Mokhtar et al. (2014) found that the TBA values of different treated samples (chitosan, rosemary extract and carnosine) TBA formation increased rapidly with storage time in the control samples. Addition of the examined antioxidants showed significant $(\mathrm{p}<0.05)$ effects compared to control during storage of beef patties. Samples containing rosemary extract and its 
combinations (ROSE, ROSE + CHI and ROSE $+\mathrm{CAR})$ together with those containing (CHI + CAR) exhibited the lowest ( $p<0.05)$ TBA values compared to those containing the individual antioxidants (CHI, CAR), a fact that indicates the occurrence of a synergistic effect. Jin et al. (2016) reported that TBA measured as the lipid oxidation value increased with storage period in treated thyme essential oil samples during storage sausages. However, the TBA value did not show any consistent trends between treated samples during storage.

Egyptian organization for standardization and quality (E.O.S., 2006) had laid down the TVB$\mathrm{N}$ level must not be more than $20 \mathrm{mg} / 100 \mathrm{~g}$ in chilled meat.

Total volatile basic-nitrogen (TVB-N) is a substance produced in the process of meat spoilage, and its content in meat was proportional to the extent of meat spoilage. So it is an important index to evaluate the degree of meat freshness Shanmei and Gan (2016). Meat raw material is the natural source of the substrate from which biogenic amines are produced. It also is the largest component of the matrix in which the decarboxylation reactions take place and any conditions that alter its nature and characteristics will influence the formation of biogenic amines (Ruiz-Capillas and Jiménez-Colmenero (2004).

Accordingly, the presence of biogenic amines can cause several problems for susceptible consumers, such as nausea, respiratory disorder, hot flushes, sweating, heart palpation, headache, bright red rash, oral burning, hypo or hypertension, whose intensity is depend on quantitative and qualitative differences. The total volatile nitrogen bases (TVBN) in meat may be increased as the days of storage increased Sayed et al. (2009).

\section{REFERENCES} (1997): The relationship at broiler breast meat odour and $\mathrm{pH}$ to shelf-life and odour development. J. poultry sci., 67: 1042-1046.

Anwer, M.; Khan, M.I.; Pasha, I.; Tariq, M. R. and Sohaib, M. (2013): Quality assessment of meat in relation to colour and muscle fiber. Pak. J. food sci., 23(2): 80-86.

Baranauskiene, R.; Venskutoni, S. P. R.; Viskelis, P. and Dambrauskiene, E. (2003): Influence of nitrogen fertilizers on the yield and composition of thyme (Thymus vulgaris). Journal of Agricultural and Food Chemistry, 51: 7751-7758.

CIE (Commission International de l'E' clairage) (1976): Official recommendations on uniform colour spaces. Colour difference equations and metric colour terms, Suppl. No. 2. CIE Publication No. 15 Colourimetry. Paris.

Devlieghere, F.; Vermeiren, L. and Debevere, J. (2004): New preservation technologies: possibilities and limitations. International Dairy Journal, 14:273-285.

Egyptian standard (ES: 63-10/2006): Methods of analysis and testing for meat and meat products. Part: 10: Determination of thiobarbituric acid (TBA).

Egyptian standard (ES: 63-9/2006): Methods of analysis and testing for meat and meat products. Part: 9: Determination of total volatile nitrogen.

Friedman, M. and V.K. Juneja (2010): Review of antimicrobial and antioxidative activities of chitosans in food. J. Food Prot., 73: 1737-1761. 
Fernández-López, J.; Pérez- Alvarez, J.A. and Aranda-Catalá, V. (2000): Effect of mincing degree on color properties in pork meat. Res. Appl., 25: 376-380.

Georgantelis, D.; Blekas, G.; Katikou, P.; Ambrosiadis, I.; Fletouris, D.J. (2007): Effect of rosemary extract, chitosan and a-tocopherol on lipid oxidation and colour stability during frozen storage of beef burgers, Meat Science, 75, 256-264.

Goy, R. C.; De Britto, D. O. and Assis, B. G. (2009): A Review of the Antimicrobial Activity of Chitosan. Polímeros: Ciência e Tecnologia, 19 (3): 241-247.

Hassan, R.; El-Kadi, S. and Sand, M. (2015): Effect of some organic acids on some fungal growth and their toxins production. International Journal of Advances in Biology, 2(1): 1-11.

ISO (21527-1:2008): Microbiology of food and animal feeding stuffs - Horizontal method for the enumeration of yeasts and mould. Part 1: Colony count technique in products with water activity greater than 0.95 .

ISO 6887 (all parts) (2017): Microbiology of food and animal feeding stuffs Preparation of test samples, initial suspension and decimal for microbiology examination.

Jay, J.M.; Loessner, J.L. and Golden, D.A. (2005): Chapter 2: Taxonomy role and significance of microorganisms in foods. Chapter 13: Food protection with chemicals and biocontrol. In: Modern Food Microbiology (edited by J.M. Jay, J.L. Loessner and D.A. Golden) (7th Ed.). New York: Springer Science and Business Media, Inc.
Jin, S. K.; Choi, J. S.; Lee, S. J.; Lee, S. Y. and Hur, S. J. (2016): Effect of Thyme and Rosemary on the quality characteristics, shelf-life, and residual nitrite content of sausages during cold storage. Korean $\mathbf{J}$ Food Sci Anim Resour., 36(5): 656664.

Jo, C.; Lee, J.W.; Lee, K.H. and Byun, M.W.(2001): Quality properties of pork sausage prepared with water soluble chitosan oligomer, Meat Science, 59: 369-375.

Karabagias, I.; Badeka, A. and Kontominas, M.G. (2011): Shelf life extension of lamb meat using thyme or oregano essential oils and modified atmosphere packaging. Meat Science, 88, 109-116.

Kocić-Tanackov, S. D. and Dimić, G. R. (2013): Antifungal activity of essential oils in the control of food-borne fungi growth and mycotoxin biosynthesis in food. Microbial pathogens and strategies for combating them: science, technology and education (A. MéndezVilas, Ed.)(C FORMATEX, 838-849.

McDougall, D.B. (1983): Changes in colour and opacity of meat, Food Chemistry, 9: 75-88.

Mokhtar, S. M.; Youssef, K. M. and Morsy, N. E. (2014): The effects of natural antioxidants on colour, lipid stability and sensory evaluation of fresh beef patties stored at $4^{\circ} \mathrm{C}$. Journal of Agroalimentary Processes and Technologies, 20(3), 282-292.

Mokhtar, S.; Mostafa, G.; Taha, R.; Eldeep, G.S.S. (2012): Effect of different starter cultures on the biogenic amines production as a critical control point in fresh fermented sausages, European 
Food Research and Technology, 235: 527-535

Park, Y.; Mi-Hyun, K.; Seong-Cheol, P., Hyeonsook, C.; Mi-Kyeong, J.; JaeWoon, N. and Kyung-Soo, H. (2008): Investigation of the Antifungal Activity and Mechanism of Action of LMWSChitosan. J. Microbiol. Biotechnol., 18(10), 1729-1734.

Rabea, E.I.; Badawy, M.E.T.; Stevens, C.V.; Smagghe, G. and Steurbaut, W. (2003): Chitosan as antimicrobial agent, applications and mode of action. Biomacromolecules, 4: 1457-1465.

Rahman, M. H.; Hossain, M. M.; Rahman, S. M. E.; Amin, M. R. and Deog-Hwan, O. (2015): Evaluation of physicochemical deterioration and lipid oxidation of beef muscle affected by freeze-thaw cycles. Korean J. Food Sci. An., 35(6):772-782.

Ruiz-Capillas, C. and Jiménez-Colmenero, F. (2004): Biogenic amines in meat and meat products. Crit. Rev. Food Sci. 44, 489-499.

Senel, S. and McClure, S.J. (2004): Potential applications of chitosan in veterinary medicine. Adv. Drug Deliv. Rev., 56: 1467-1480.

Sayed, M.; Seyed, S.S.; Javad, A.; Mohamad, A. and Zakaria, V. (2009): Effect of processing factors on biogenic amines production in Iranian white brine cheese. Research Journal of Biological Sciences 4 (1): 23-38.

Shanmei, L. and Gan, L. (2016): Determination of Total Volatile Basic Nitrogen (TVB-N) Content in Beef by Hyperspectral Imaging Technique. MATEC Web of Conferences volume 61, article no. 06007: 5.
Youn, S.K.; Park, S.M.; Kim, Y.J. and Ahn, D.H.(1999): Effect on storage property and quality in meat sausage by added chitosan, Journal of chitin and chitosan,4:189-195.

Zahran, D.A. (2015): Effectiveness of Gamma Irradiated Chitosan for Fresh Meat Preservation. Arab Journal of Nuclear Science and Applications, 48(3): 143152. 